\title{
Fréquence des rétractations d'articles scientifiques : ce que les études nous apprennent en éducation, sciences humaines et sciences sociales
}

Roy-Vallières

Université du Québec à Montréal

\section{Résumé}

Cette étude souhaitait déterminer la fréquence des articles portant sur les rétractations dans les différents domaines scientifiques et explorer les raisons qui poussent les chercheurs à s'y intéresser. Pour ce faire, une revue de littérature non systématique a été réalisée. Les résultats montrent que $70 \%$ des articles sur les rétractations se retrouvent en sciences naturelles et médicales, et que les raisons poussant les chercheurs à s'intéresser au phénomène sont partagées dans tous les domaines. Des spécificités liées aux champs de recherche sont toutefois soulevées pour expliquer la quantité réduite de rétractations en sciences humaines et sociales, et spécifiquement en éducation.

Mots-clés : rétractation d'articles, revue de littérature, raisons et intérêts, domaines scientifiques 


\begin{abstract}
This study aimed at establishing prevalence of retraction articles in various scientific fields and exploring reasons why researchers are interested in the phenomenon. To do this, a non-systematic literature review was carried out. Results show that $70 \%$ of retraction articles pertain to the natural and medical sciences and that the reasons leading researchers to take an interest in retractions are shared in all science fields. However, specificities of research fields are raised to explain the reduced number of retraction studies in the human and social sciences domain, and specifically in education.
\end{abstract}

Keywords: article retraction, literature review, reasons and interest, scientific fields 


\section{Introduction}

Dans les dernières décennies, l'attention portée aux pratiques éthiques en recherche, particulièrement au sein des publications scientifiques, s'est décuplée. Il faut soulever notamment le travail de Retraction Watch, un blogue doublé d'une base de données dont la mission consiste à promouvoir la transparence de la recherche scientifique en médiatisant les articles rétractés (Marcus et Oransky, 2020). De la même façon, le Committee on Publication Ethics (COPE) s'est dédié, depuis une vingtaine d'années, à l'élaboration de lignes directrices sur l'éthique de la publication, tant pour les auteurs que pour les éditeurs (COPE, 2021).

Cet intérêt marqué pourrait être lié aux résultats d'études récentes qui laissent penser que les instances d'inconduites en recherche connaissent une augmentation dans les dernières années (Fanelli, 2009 ; Nature, 2013). Effectivement, une métaanalyse de Fanelli (2009) a rapporté qu'environ $2 \%$ des chercheurs questionnés dans divers sondages avaient admis avoir fraudé au moins une fois, c'est-à-dire avoir falsifié ou modifié des données. Le tiers reconnaissait également avoir réalisé d'autres types d'inconduites, tout en rapportant avoir été témoins d'inconduites chez leurs collègues (Fanelli, 2009). Plus récemment, l'Office of Research Integrity rapportait une augmentation des allégations de fraude de $216 \%$ depuis 2001 (Nature, 2013). Bien que cette augmentation puisse être liée à l'amélioration des outils et des pratiques de détection de la fraude (Basken, 2012 ; Bonnet et Samama, 2012), ces données donnent à penser que les cas d'inconduites en recherche ne seraient pas de simples exceptions.

\section{Une problématique particulièrement sensible en éducation}

Chez l'être humain, la démarche scientifique est la quête de la connaissance qui mène à la compréhension des lois qui forment notre monde (Cokol et al., 2008). La littérature scientifique constitue, en quelque sorte, un recueil historique témoignant du cheminement de nos connaissances (Coudert, 2019 ; Steen, 2011). Dans une société de plus en plus mondialisée, la diffusion de ces connaissances est cruciale pour assurer un avancement efficace de la science et des pratiques (Palla et al., 2020 ; Rosenkrantz, 2016).

Alors qu'on s'attendrait à ce que les enseignants et les décideurs en éducation s'approprient les connaissances disséminées par les chercheurs pour améliorer 
promptement les pratiques et les politiques (Randi et Corno, 2007), des recherches ont montré que le transfert des connaissances scientifiques s'avère problématique (Hille, 2011). Effectivement, les acteurs de l'éducation baseraient davantage leurs pratiques sur des courants de pensée et des consensus professionnels que sur les avancées de la recherche (U.S. Department of Education et al., 2002). Les résultats des études scientifiques en éducation sont ainsi peu mobilisés en pratique. Considérant qu'un transfert de connaissances réussi s'appuie sur la confiance du public et des chercheurs eux-mêmes envers la science (Aliukonis et al., 2020 ; Nogueira et al., 2017), l'augmentation des inconduites scientifiques risque de venir ébranler cette confiance et de réduire davantage les probabilités que les découvertes en recherche soutiennent les pratiques.

Par ailleurs, la fraude engendre un gaspillage de ressources dans les milieux scientifiques (Decullier et al., 2012). Elle utilise l'argent des subventions obtenues, ainsi que le temps du personnel de recherche et des participants, sans toutefois permettre l'avancement des connaissances scientifiques (Lambert-Chan, 2013 ; Mongeon et Larivière, 2016). Bien que pertinente dans tous les champs de recherche, cette conséquence revêt une importance particulière en sciences humaines, notamment en éducation. Effectivement, les institutions subventionnaires des chercheurs en sciences humaines proviennent principalement du domaine public et sont souvent plus limitées dans leurs capacités de financement (Benneworth et Jongbloed, 2010). Le gaspillage des fonds octroyés a ainsi plus d'impact sur l'avancement des recherches des autres chercheurs et contribue à la dévalorisation de la recherche en éducation.

Finalement, il convient de mentionner l'impact de la fraude en recherche sur les participants. Les acteurs du champ de la médecine et des sciences de la santé dénoncent particulièrement cet impact dans un contexte où les participants peuvent courir des risques inutiles, voire mettre leur vie en danger, par exemple en recevant une trop faible quantité d'antidouleurs (Decullier et al., 2013 ; Steen, 2012). Cela est sans compter que les participants d'études s'inspirant de recherches frauduleuses sont aussi potentiellement à risques. Bien qu'il soit improbable qu'une étude dans le champ de l'éducation mette en danger la vie de ses participants, il faut considérer l'impact que pourraient avoir des recherches frauduleuses sur le cheminement ultérieur des participants. Par exemple, les études réalisées lors de la période de la petite enfance pourraient avoir un effet sur la réussite éducative future des participants, puisque le cerveau tend à se modifier en 
fonction des expériences vécues (Simard et al., 2013 ; Yoshikawa et al., 2013). En ce sens, il convient de mettre en place des dispositifs permettant de contrer la fraude et les inconduites en recherche, diminuant du même coup leurs retombées négatives.

\section{Prévention de la fraude}

Divers auteurs se sont ainsi penchés sur les solutions qui pourraient limiter la fraude et les inconduites. Quatre domaines d'intérêt semblent avoir retenu l'attention des chercheurs pour la réduction de la fraude. Premièrement, la culture scientifique elle-même pourrait être modifiée pour décourager les pratiques frauduleuses. Notamment, plusieurs chercheurs suggèrent d'améliorer l'éducation aux pratiques éthiques (p. ex., Fanelli et al., 2015 ; Hu et al., 2019 ; Lei et Zhang, 2018). En effet, certaines études ont rapporté que bien des chercheurs et des étudiants comprennent mal ou ignorent quelles sont les pratiques considérées comme « éthiques », ainsi que les règlements ou les sanctions liés aux inconduites (Aliukonis et al., 2020 ; Decullier et al., 2012). Ainsi, l'offre de cours sur l'éthique en recherche, le partage d'informations sur ce même sujet dans les congrès de chercheurs et la promotion de la vigilance face aux résultats des études seraient des avenues intéressantes à explorer pour assurer des pratiques intègres chez les chercheurs.

Deuxièmement, d'autres scientifiques soulignent que les établissements universitaires doivent contribuer à la réduction des instances de fraude. On pense notamment à la mise en place de structures ou de politiques pour décourager les inconduites en recherche et gérer les allégations ou les cas de fraude avérés (Decullier et al., 2012 ; Sox et Rennie, 2006). D'autres dispositifs soutenus par la recherche concernent la mise en place d'évaluations ou d'auto-évaluations des conduites éthiques chez les chercheurs (Steneck, 2006), le soutien aux équipes de recherche dans les disputes d'auteurs (Aliukonis et al., 2020), la facilitation des démarches de déclaration de la fraude (Nath et al., 2006) et une évaluation du potentiel des chercheurs plus axée sur la qualité des publications que sur leur quantité (Lee et Schrank, 2010 ; Lei et Zhang, 2018 ; Marcus et Oransky, 2011).

Troisièmement, les équipes de recherche seraient en mesure d'établir des pratiques favorisant l'intégrité chez leurs membres. Par exemple, l'utilisation d'un contrat standardisé décrivant le rôle de chaque coauteur dans la production d'un manuscrit permettrait d'assurer l'accord de tous avant la publication (Bonnet et Samama, 2012). De 
la même façon, une équipe de recherche encourageant l'inscription des essais cliniques sur des sites Internet gouvernementaux et mettant en place un environnement d'échange ouvert, critique et transparent favoriserait l'adoption de bonnes pratiques chez ses chercheurs membres (Decullier et al., 2012 ; Fanelli et al., 2015 ; Lee et Schrank, 2010).

Finalement, les éditeurs de revues scientifiques se voient aussi encouragés à améliorer leurs pratiques, considérant que la relecture par les pairs fait actuellement l'objet de plusieurs critiques (Basken, 2012 ; Lei et Zhang, 2018). Les chercheurs soulèvent effectivement plusieurs lacunes, nommément l'impossibilité de détecter la fraude sans l'accès aux données brutes, et le nombre réduit d'experts dans certains domaines qui empêche une révision adéquate dans un délai raisonnable au vu de l'importante quantité de manuscrits soumis. L'application de règlements obligeant l'annexion du certificat d'éthique au manuscrit soumis, la déclaration des conflits d'intérêts et une utilisation plus étendue des logiciels de détection de similitudes pourraient aider à vérifier l'intégrité des résultats (Bonnet et Samama, 2012). Toutefois, la rétractation d'articles demeure l'ultime recours des éditeurs et de la science pour corriger la documentation scientifique.

En effet, bien que les solutions présentées précédemment existent déjà dans la littérature, les pratiques en matière d'intégrité et de prévision de la fraude sont disparates, voire inexistantes en certains endroits (George, 2016 ; Van Noorden, 2011 ; Williams et Wager, 2013). Dans les pays nord-américains, on favorise l'éducation à l'intégrité scientifique, mais peu de données existent quant à la véritable efficacité de ces pratiques (Funk et al., 2007). Pour ce qui est des établissements universitaires, peu d'entre eux possèdent actuellement des réglementations et affichent des sanctions claires quant à la fraude scientifique. En outre, Marcus et Oransky (2011) ont soulevé la difficulté pour ces établissements de trouver des mesures fiables autres que la quantité d'articles publiés pour évaluer la productivité des chercheurs. Au niveau des équipes de recherche, il est tout à fait probable que la majorité d'entre elles n'utilisent pas de contrats ou toute autre forme de réglementation claire qui viendrait spécifier les normes d'éthique et contribuer à réduire les conflits d'auteurs. Finalement, les pratiques des éditeurs en matière d'intégrité dans les publications sont elles aussi extrêmement variées, particulièrement en ce qui concerne le format de leurs avis indiquant des modifications apportées à un article (Decullier et al., 2012 ; Marcus et Oransky, 2011 ; Steen, 2011). Il existe donc certaines solutions en amont qui permettraient de limiter la fraude, mais les limites exposées 
ci-dessus montrent que celles-ci sont encore à développer. En ce sens, la rétractation d'articles demeure le dernier recours de la science pour se purger elle-même des contributions erronées.

\section{Rétractations d'articles}

La rétractation d'article concerne le fait de retirer un article publié de la littérature scientifique en raison de résultats qui ont été invalidés et qui ne peuvent ainsi plus faire partie du répertoire des connaissances scientifiques humaines (Li et al., 2018 ; Williams et Wager, 2013). Elle constitue donc un mécanisme d'autocorrection de la littérature scientifique et ne vise pas à punir les auteurs, mais plutôt à purger le bassin des connaissances des erreurs (Fang et Casadevall, 2011 ; Rubbo et al., 2019). Plusieurs la considèrent également comme la sanction la plus sévère qui puisse être appliquée à un article (Chen et al., 2013 ; Steen, 2011 ; Williams et Wager, 2013). Cependant, la rétractation d'article n'implique généralement pas la disparition totale de l'article du registre scientifique, ce qui nuirait à la transparence (Decullier et al., 2013 ; Peterson, 2013). Il s'agit plutôt d'apposer un avis de rétractation à l'article, sous forme de filigrane, afin d'avertir les lecteurs potentiels que celui-ci contient des informations erronées (Azoulay et al., 2017 ; Rosenkrantz, 2016).

Les rétractations d'articles scientifiques ont reçu beaucoup d'attention dans les dernières années (Bilbrey et al., 2014). L’intérêt des médias et des chercheurs face à ce sujet serait lié à l'apparente augmentation de la fréquence des rétractations dans le temps (Grieneisen et Zhang, 2012 ; Mena et al., 2019). Des chercheurs (p. ex., Chen et al., 2013 ; Decullier et al., 2013 ; Stretton et al., 2012) auraient effectivement rapporté une augmentation significative des rétractations depuis le début du siècle. Selon Decullier et al. (2013), « The number of retractions in journals covered by the Science Citation Index Expanded has increased 20 times i.e. a tenfold increase since there has been a twofold increase in articles production between 1990 and $2008 »$ (p. 1). Bien que cette augmentation semble être connue et acceptée dans la communauté scientifique, les causes de celle-ci sont encore nébuleuses (Azoulay et al., 2017 ; Van Noorden, 2011). En ce sens, plusieurs études ont entrepris de circonscrire le phénomène.

On remarque cependant une tendance. Les études s'intéressant aux rétractations d'articles scientifiques se concentrent majoritairement dans le domaine des sciences de 
la santé (Grieneisen et Zhang, 2012), bien que l'on puisse nommer quelques exceptions (p. ex., Cox et al., 2018 ; Karabag et Berggren, 2016 ; Rubbo et al., 2019). Pourtant, les problématiques soulevées plus haut montrent que la fraude a un impact négatif important sur la pratique, les participants et les avancées de la science, et ce, particulièrement dans le domaine de l'éducation. En outre, différentes sources, comme Retraction Watch (Marcus et Oransky, 2020) et les études de Lu et al. (2013) et de Grieneisen et Zhang (2012), ont montré que les rétractations ne se limitent effectivement pas au domaine de la santé. Toutefois, mis à part dans les sciences de la santé, on ne retrouve aucune étude systématique des rétractations dans les autres domaines de recherche (Coudert, 2019). Le nombre de rétractations et, par le fait même, l'importance des incidences de fraude dans les autres champs de recherche demeurent ainsi incertains.

Considérant les impacts négatifs de la fraude en éducation et la nécessité de données probantes pour encourager davantage les méthodes de prévention, la présente étude vise à déterminer si les rétractations sont effectivement moins explorées en sciences humaines, et plus spécifiquement en éducation, en réalisant une revue de littérature sur les rétractations dans les divers domaines scientifiques. Elle tente également de comprendre pourquoi ce processus d'autocorrection de la science est plus ou moins présent en éducation en avançant diverses hypothèses liées aux spécificités des domaines de recherche. De façon générale, les résultats de cette étude pourront contribuer à informer les chercheurs sur la fréquence des rétractations dans leur domaine spécifique de recherche. En éducation, la revue de littérature permettra d'informer sur les raisons pour lesquelles les rétractations sont plus ou moins présentes et, par la suite, d'identifier des pistes d'amélioration permettant d'assurer un meilleur contrôle de la conduite éthique dans les publications du domaine. En retour, ces propositions contribueront à diminuer les retombées néfastes de la fraude dans le champ de l'éducation. La section qui suit décrit la méthode utilisée pour répondre aux objectifs de la recherche. 


\section{Méthode}

\section{Recensement des articles scientifiques}

La première étape du processus d'analyse consistait à identifier des articles scientifiques provenant de revues avec processus d'évaluation par les pairs et pertinents par rapport aux objectifs de la recherche. La recension a été limitée à des articles évalués par les pairs dans le but d'assurer une certaine consistance dans la qualité des propos évalués. Elle visait par ailleurs l'ensemble des articles en langue française ou anglaise abordant les rétractations dans un domaine de recherche et publiés jusqu'en juillet 2020. L'ensemble des bases de données accessibles grâce à la bibliothèque de l'Université du Québec à Montréal (p. ex., Business Source Complete, Erudit, JSTOR, PsychINFO, PubMed, SAGE Journals, SCOPUS) ainsi que le moteur de recherche Google Scholar ont été consultés pour générer une liste multidisciplinaire d'articles. Les mots-clés « retracted articles in », " article retraction in », « article withdrawal in », " retraction of papers in », « articles retirés en » et « rétractations d'articles en » ont été utilisés pour chercher dans les bases de données et adjoints de termes liés aux quatre grandes branches de la science retenues : 1) sciences pures (p. ex., algèbre, mathématiques, géométrie, informatique, logique, topologie) ; 2) sciences naturelles et médicales (p. ex., sciences de la terre, de l'univers, de la matière [chimie/physique], de la vie [biologie, écologie, génétique, physiologie, médecine, neurosciences]) ; 3) sciences humaines et sociales (p. ex., psychologie, linguistique, histoire, sociologie, éducation, économie, politique) ; et 4) sciences appliquées (p. ex., aéronautique, agronomie, électronique, nanotechnologie, ingénierie).

Chaque mot-clé a généré un nombre important de résultats. Les articles ressortant des résultats de recherche n'étaient pas retenus s'ils avaient déjà été recensés à partir d'une autre base de données. Les articles provenant de revues sans processus d'évaluation par les pairs ou ne comprenant pas de résultats de recherche empiriques étaient également exclus. Finalement, les articles utilisant le terme « rétractations » sous un autre sens que celui décrit dans la présente étude (p. ex., au sens médical lié à l'asthme) n'ont pas été retenus. À l'inverse, certains articles respectant les critères mentionnés plus haut, mais ne provenant pas des recherches dans les bases de données, ont été ajoutés à la recension. 
Ces articles ont été choisis à partir des références personnelles de l'auteure, ainsi qu'à partir des références bibliographiques des articles précédemment recensés. La méthode décrite ci-haut a permis de recenser 122 articles abordant les rétractations d'articles dans la littérature scientifique.

\section{Analyse des articles scientifiques}

Des copies électroniques de chaque article ont été obtenues pour favoriser une analyse à la fois quantitative et qualitative des propos. L'analyse des articles via le logiciel NVivo 12 (QSR International, 2020) a permis d'identifier les segments de texte pertinents et de les associer à des catégories communes, tout en permettant de sauvegarder les fichiers récoltés au même endroit.

D'abord, afin de répondre à l'objectif plus quantitatif de déterminer si les rétractations d'article sont moins explorées en sciences humaines, et spécifiquement en éducation, l'auteure a examiné les titres et les résumés de chaque article pour faire ressortir des mots-clés permettant de les classer dans l'une des quatre branches de la science retenues (sciences pures, sciences naturelles et médicales, sciences humaines et sociales, sciences appliquées). Ces mots-clés pouvaient référer directement à l'une des quatre branches ou spécifier un champ de recherche en particulier. Pour certains articles, il a également été nécessaire d'analyser les parties de l'introduction ou de la problématique, puisque le titre ou le résumé ne s'avéraient pas suffisamment informatifs. Au cours de l'analyse, une cinquième catégorie a dû être ajoutée pour classifier les articles, soit la catégorie « multidisciplinaire ». En effet, l'analyse par mots-clés a révélé plusieurs articles qui avaient réalisé une recension des rétractations dans plus d'un domaine scientifique ou qui ne spécifiaient pas la branche de la science liée aux résultats présentés.

Ensuite a eu lieu une analyse inductive des raisons pour lesquelles le processus de rétractation d'articles scientifiques est moins présent en éducation, et plus largement en sciences humaines, selon la méthode décrite par Blais et Martineau (2006), inspirée de Thomas (2006). Après avoir regroupé l'ensemble des textes recensés dans le logiciel NVivo, l'auteure a procédé à une lecture approfondie de ceux-ci, chaque texte ayant été révisé à plusieurs reprises. L'analyse des propos d'une première dizaine d'articles a permis de procéder à l'identification de segments de textes significatifs au regard des 
objectifs de recherche et de les classer dans des catégories préliminaires. Ces catégories ont par la suite évolué de pair avec la progression de la lecture des autres éléments du corpus. Au bout du compte, trois questions, découlant de l'objectif de comprendre pourquoi l'on retrouve moins de rétractations en éducation, ont guidé l'analyse des articles recensés :

1. Quelles sont les raisons qui poussent les chercheurs à étudier les rétractations dans leur domaine?

2. Parmi ces raisons, lesquelles appartiennent aux sciences humaines et sociales?

3. En quoi les particularités des différents domaines scientifiques ont-elles un impact sur la fréquence des rétractations ou sur l'intérêt porté à ces rétractations?

La section suivante présente les résultats tirés de l'analyse des données recueillies.

\section{Résultats}

\section{Fréquence des études de rétractations d'articles scientifiques selon les domaines}

La méthodologie décrite dans le présent article a permis de recenser 122 écrits abordant les rétractations d'articles dans la littérature scientifique. De ce nombre, 85 (69,7\%) publications concernaient les rétractations d'articles dans les sciences naturelles et médicales ; 11 (9\%) se rapportaient aux rétractations dans les sciences humaines et sociales; un seul article s'y rapportait dans chaque domaine pour les sciences pures ( $1 \%$ ) et appliquées ( $1 \%$ ) ; enfin, 24 articles (19,7\%) ont été classés dans la catégorie « multidisciplinaire ». Le Tableau 1 présente les résultats descriptifs de cette revue de littérature, incluant les domaines abordés par les articles. Il est intéressant de constater qu'aucun article du corpus ne portait spécifiquement sur le domaine de l'éducation. 


\section{Tableau 1}

Analyse descriptive des articles portant sur les rétractations dans la littérature scientifique

\begin{tabular}{|c|c|c|}
\hline Branches de la science & Domaines spécifiques abordés & $\begin{array}{l}\text { Nre d'articles } \\
\text { recensés }\end{array}$ \\
\hline $\begin{array}{l}\text { 1. Sciences naturelles et } \\
\text { médicales }\end{array}$ & $\begin{array}{l}\text { Anesthésiologie ; biologie ; biologie moléculaire ; biomé- } \\
\text { decine ; cancer ; chimie ; chirurgie ; chirurgie plastique ; } \\
\text { génie chimique ; infectiologie ; médecine ; médecine de } \\
\text { soins intensifs ; médecine d’urgence ; neurochirurgie ; } \\
\text { obstétrique et gynécologie ; odontologie ; orthopédie ; } \\
\text { pharmacologie ; radiologie ; radio-oncologie ; réhabilita- } \\
\text { tion ; santé mentale ; soins infirmiers et sages-femmes ; } \\
\text { sciences des matériaux ; urologie. }\end{array}$ & 85 \\
\hline $\begin{array}{l}\text { 2. Sciences humaines et } \\
\text { sociales }\end{array}$ & $\begin{array}{l}\text { Administration ; arts ; bibliothéconomie ; comptabilité ; } \\
\text { économie ; gestion ; psychologie ; tourisme. }\end{array}$ & 11 \\
\hline 3. Sciences pures & Informatique. & 1 \\
\hline 4. Sciences appliquées & Ingénierie. & 1 \\
\hline 5. Multidisciplinaire & Tous. & 24 \\
\hline
\end{tabular}

\section{Raisons soutenant la fréquence des études de rétractations}

L'analyse se tourne maintenant vers le deuxième objectif de cette recherche, concernant les raisons pour lesquelles les recensions de rétractations sont moins présentes en éducation. Considérant qu'aucun article abordant les rétractations dans le domaine de l'éducation n'a pu être recensé, l'analyse concernera plus largement les sciences humaines et sociales. Les paragraphes suivants présentent les résultats liés aux trois questions qui ont guidé la recherche.

\section{Quelles sont les raisons qui poussent les chercheurs à étudier les rétractations}

dans leur domaine? L'analyse du corpus a permis l'extraction de huit raisons principales de s'intéresser aux rétractations d'articles. Une grande proportion d'auteurs (40,1\%) a soulevé la nécessité d'étudier les rétractations en raison de leurs impacts négatifs sur la recherche et la pratique. L'exploration des rétractations permettrait ainsi de sonder la partie visible de la fraude scientifique dans le but de la contrer. D'autres raisons évoquées fréquemment concernent : l'importante médiatisation de certains cas de fraude $(28,7 \%)$, qui attirent l'attention des chercheurs et du public sur le phénomène des rétractations ; 
le nombre annuel élevé de publications dans certains domaines de recherche très actifs $(21,3 \%)$, qui rend difficile la vérification minutieuse de chaque article ; et le ratio de rétractations/publications totales relativement élevé dans certaines disciplines $(24,6$ $\%$ ), qui pourrait être le signe d'une présence plus importante de fraude. D'une façon moins importante, certains chercheurs ont soulevé l'absence de recherches antérieures sur le sujet $(10,7 \%)$; l'importance d'étudier les rétractations pour assurer la véracité des informations disponibles ( $9 \%$ ) ; et la présence d'une culture encourageant la fraude dans certains champs de recherche, phénomène qui doit être étudié pour parvenir à le contrer (2,4\%). À l'opposé, des auteurs ont mentionné la difficulté de s'intéresser aux rétractations dans certains domaines scientifiques étant donné l'absence de critères de recherche appropriés dans les bases de données (18,9\%), qu'ils qualifient d'ailleurs d'incomplètes. Il est finalement intéressant de noter que les auteurs de 23 publications recensées n'ont pas nommé les raisons qui les poussaient à s'intéresser aux rétractations d'articles scientifiques, dont ceux de l'unique publication identifiée en sciences appliquées.

\section{Parmi ces raisons, lesquelles appartiennent aux sciences humaines et sociales?}

Le Tableau 2 permet de représenter numériquement la répartition des raisons justifiant l'intérêt des chercheurs quant aux rétractations d'articles scientifiques. Il est possible d'en ressortir qu'aucune raison n'appartient uniquement au domaine des sciences humaines et sociales. Effectivement, les auteurs en sciences naturelles et médicales, en sciences pures et dans les articles multidisciplinaires ont soulevé les mêmes raisons que ceux en sciences humaines et sociales. Par ailleurs, l'unique raison non soulevée par les chercheurs en sciences humaines et sociales concerne la culture du domaine encourageant la fraude.

\section{Tableau 2}

Raisons de s'intéresser aux rétractations en fonction des domaines de recherche

\begin{tabular}{lccccc}
\hline & \multicolumn{4}{c}{ Domaines de recherche } \\
\cline { 2 - 6 } \multicolumn{1}{c}{ Raisons } & $\begin{array}{c}\text { Sc. } \\
\text { naturelles } \\
\text { et } \\
\text { médicales }\end{array}$ & $\begin{array}{c}\text { Sc. } \\
\text { humaines } \\
\text { et sociales }\end{array}$ & Sc. pures & $\begin{array}{c}\text { Sc. } \\
\text { appliquées }\end{array}$ & Multi. \\
\hline $\begin{array}{l}\text { Impacts négatifs sur la recherche et la } \\
\text { pratique }\end{array}$ & 37 & 4 & 1 & - & 7 \\
\end{tabular}




\begin{tabular}{lccccc}
\hline & \multicolumn{5}{c}{ Domaines de recherche } \\
\cline { 2 - 6 } \multicolumn{1}{c}{ Raisons } & $\begin{array}{c}\text { Sc. } \\
\text { naturelles } \\
\text { et } \\
\text { médicales }\end{array}$ & $\begin{array}{c}\text { Sc. } \\
\text { humaines } \\
\text { et sociales }\end{array}$ & Sc. pures & $\begin{array}{c}\text { Sc. } \\
\text { appliquées }\end{array}$ & Multi. \\
\hline Médiatisation des cas de fraude & 19 & 7 & - & - & 9 \\
Nombre annuel élevé de publications & 19 & 4 & - & - & 3 \\
$\begin{array}{l}\text { Présence/Absence d'un critère de } \\
\text { recherche dans les banques de données }\end{array}$ & 16 & 4 & - & - & 3 \\
$\begin{array}{l}\text { Ratio rétractations/publications élevé } \\
\text { Absence de recherches antérieures }\end{array}$ & 15 & 7 & - & - & 8 \\
Culture encourageant la fraude & 7 & 2 & 1 & - & 3 \\
$\begin{array}{l}\text { Assurer la véracité des informations } \\
\text { disponibles }\end{array}$ & 1 & - & - & - & - \\
\hline
\end{tabular}

En quoi les particularités des différents domaines scientifiques ont-elles un impact sur la fréquence des rétractations ou sur l'intérêt porté à ces rétractations? Pour répondre à cette question, il était nécessaire de faire ressortir les différences qui existent entre les grands domaines de recherche. Certains propos retenus dans le cadre des analyses liées aux deux questions précédentes permettent d'apporter des éléments de réponse à cette question. Dans le domaine des sciences naturelles et médicales, 36,1\% des auteurs font remarquer que les praticiens du domaine s'appuient hautement sur les résultats de la recherche scientifique pour orienter leurs pratiques (Bakker et Riegelman, 2018 ; Budd et al., 2011). Ceci constitue une différence par rapport aux informations rapportées dans l'introduction quant à la tendance des praticiens en éducation à baser davantage leur pratique sur des courants de pensée. Par ailleurs, un certain nombre d'articles $(16,4 \%)$ du corpus soulignent que le nombre de rétractations ainsi que le ratio de rétractations/publications totales sont plus élevés en sciences naturelles et médicales que dans les autres domaines. Les champs de recherche en sciences naturelles et médicales auraient donc comme particularité d'être plus à risques, de manière relative et absolue, d'offrir des connaissances erronées nécessitant le retrait d'un article pour corriger le tir (Aspura et al., 2018 ; Grieneisen et Zhang, 2012 ; Shuai et al., 2017).

Il est également possible de soulever quelques particularités du champ des sciences humaines et sociales nommées par les auteurs du corpus pouvant avoir un impact sur la fréquence ou l'intérêt porté aux rétractations dans ce domaine. Notamment, 18,9\% des auteurs du corpus mentionnent qu'il est difficile de s'intéresser aux rétractations hors 
des sciences médicales, puisqu'il n'existe aucune base de données complète regroupant l'ensemble, ou une majorité, des revues liées aux divers champs de recherche. La recherche d'articles rétractés demande ainsi plus de temps et de ressources, ce qui peut décourager les chercheurs s'y intéressant (Karabag et Berggren, 2012). Par ailleurs, 4,1 $\%$ des auteurs ont mentionné des habitudes de reproduction d'études qui diffèrent en sciences naturelles et en sciences sociales. Le fait que la reproduction d'études existantes en sciences humaines et sociales soit rare contribuerait au nombre réduit et à l'attention moindre associés aux rétractations dans ce domaine ; en revanche, la reproduction rapide et intensive des recherches spécialisées en sciences naturelles et médicales permettrait de vérifier plus rapidement la validité des informations diffusées (Cox et al., 2018 ; Craig et al., 2020 ; Madlock-Brown et Eichmann, 2015). Or, une autre spécificité des sciences humaines et sociales entre en jeu dans les pratiques de reproduction des études. Dans un article du corpus étudié, Berland et al. (2015) soulignent « qu'il est moins facile de remettre en cause les conclusions des recherches en sciences "inexactes" que celles des recherches en sciences “exactes” » (p. 9). Les méthodologies qualitatives, typiquement utilisées en sciences sociales, sont effectivement plus complexes à reproduire de manière exacte que les méthodologies quantitatives, puisqu'elles s'appuient grandement sur le jugement du chercheur (Alrawadieh et Zareer, 2019).

\section{Discussion}

La présente étude visait à déterminer si les rétractations sont moins explorées en sciences humaines, et plus spécifiquement en éducation, par une revue de littérature non systématique transversale aux différents champs de recherche. Elle cherchait aussi à comprendre pourquoi ce processus d'autocorrection de la science est plus ou moins présent en éducation en avançant des hypothèses liées aux spécificités des domaines de recherche.

En premier lieu, il semble pertinent de mentionner que la présente recherche prête un appui aux propos de Grieneisen et Zhang (2012) et d'autres auteurs (p. ex., Alrawadieh et Zareer, 2019 ; Tchao, 2014) concernant la prépondérance des études en rétractations dans le domaine de la santé. Effectivement, les résultats de la présente revue de littérature ont montré que près de $70 \%$ des études ayant exploré les rétractations 
d'articles scientifiques appartiennent au domaine général des sciences naturelles et médicales. Elle contribue également à attirer l'attention sur la rareté des études sur le sujet dans les domaines des sciences pures et des sciences appliquées, ainsi que leur absence marquée en éducation.

En ce qui concerne les raisons qui soutiennent la fréquence des rétractations et de l'intérêt pour celles-ci dans les différents domaines, le corpus permet l'extraction de huit justifications principales. Avec raison, l'argument le plus souvent rapporté par les auteurs pour s'intéresser aux rétractations concerne l'importance d'étudier le phénomène pour limiter ses impacts négatifs sur la recherche et la pratique. Cette raison est en cohérence avec les propos présentés en introduction quant à l'influence de la fraude sur le transfert des connaissances, l'utilisation des ressources financières et la vie des participants (Aliukonis et al., 2020 ; Decullier et al., 2013 ; Mongeon et Larivière, 2016). En plus de justifier la pertinence des études portant sur les rétractations en sciences naturelles et sociales, ce constat invite à poursuivre les efforts des chercheurs pour étudier le phénomène en sciences humaines et sociales, et révèle toute son importance dans le domaine spécifique de l'éducation.

Les résultats permettent aussi de constater que la visibilité des rétractations amène les chercheurs à s'intéresser davantage au phénomène. Les raisons mentionnées, qui concernent la médiatisation des cas de fraude, le nombre absolu ainsi que le ratio rétractations/publications totales plus élevé dans les domaines des sciences naturelles et médicales, montrent que plus le partage d'informations sur les rétractations est commun et efficace, plus les chercheurs auront tendance à s'impliquer dans l'exploration et l'amélioration de ce mécanisme d'autocorrection de la science. Dans un contexte où plusieurs auteurs qualifient les mécanismes de détection de la fraude d'absents ou d'inefficaces, et ce, particulièrement en sciences humaines et sociales (Alrawadieh et Zareer, 2019 ; Berland et al., 2015 ; Da Silva et Bornemann-Cimenti, 2017 ; Karabag et Berggren, 2016), il semble adéquat de suggérer l'implantation de pratiques plus uniformes en lien avec les rétractations chez les éditeurs de ces domaines de recherche, notamment en éducation.

En ce qui concerne les raisons spécifiques au domaine de l'éducation, puisqu'aucun article étudiant les rétractations n'a pu être recensé dans ce domaine, les conclusions se rapportent aux sciences humaines et sociales d'une façon générale. Les résultats montrent ainsi que les inquiétudes des chercheurs les poussant à étudier 
le phénomène des rétractations sont partagées dans tous les domaines de recherche ciblés. Toutefois, un résultat intéressant concerne l'absence de justification en lien avec une culture scientifique encourageant la fraude dans le champ des sciences humaines et sociales. Une explication facile de ce phénomène consisterait à déclarer que les chercheurs des domaines humains et sociaux sont plus intègres et agissent de façon plus éthique. Or, comme le soulignent Berland et al. (2015), il est difficile de déterminer si la culture scientifique diffère réellement entre les sciences naturelles et médicales et les sciences humaines et sociales, alors qu'il est tout à fait possible que les rétractations soient simplement moins visibles dans certains domaines, pour les raisons exposées plus haut. En ce sens, il importe de rester vigilants et d'établir des directives éthiques claires en ce qui concerne les publications scientifiques, peu importe le champ de recherche. Il relève également de la responsabilité des universités et des chercheurs actuels d'informer les étudiants cheminant en recherche des pratiques éthiques attendues.

Finalement, au niveau des spécificités des domaines scientifiques pouvant influencer la fréquence ou l'intérêt porté aux rétractations, il est possible d'observer des contrastes importants entre le domaine des sciences naturelles et médicales, et celui des sciences humaines et sociales. Les habitudes d'utilisation des connaissances scientifiques par les praticiens constituent une différence notable, étant donné qu'une conclusion erronée en sciences naturelles risque d'avoir des retombées négatives beaucoup plus rapides et importantes sur le public qu'en éducation ou en sciences humaines et sociales. Toutefois, il s'agit là encore d'une distinction entre les domaines : les résultats des sciences naturelles et médicales sont typiquement de nature quantitative, et donc plus simples à analyser et à reproduire, alors qu'une quantité importante de chercheurs en éducation adoptent des méthodologies qualitatives s'appuyant sur l'interprétation de la réalité au travers des yeux du chercheur, du praticien ou des participants (parfois même des enfants).

Alors que le domaine des sciences humaines pourrait gagner à s'ouvrir à la pratique de reproduction des études, il est nécessaire de contextualiser les résultats des études du domaine en fonction du cadre méthodologique choisi par le chercheur. Une étude réalisée dans un contexte spécifique et faisant appel au fonctionnement interne de la pensée humaine présente un niveau de difficulté très élevé quant à la reproduction et à la vérification des résultats. Il ne s'agit toutefois pas nécessairement d'une « mauvaise » étude. Dans ces domaines où les connaissances scientifiques peuvent plus difficilement 
être confirmées, il importe donc de redoubler d'efforts en amont pour éviter que les pratiques frauduleuses ne se propagent. On retiendra notamment l'offre de cours sur l'éthique de la recherche dès le début des études supérieures, la mise en place de mécanismes pour aider à gérer les inconduites au sein des établissements universitaires et des équipes de recherche, et le suivi de directives communes de lutte contre la fraude pour l'ensemble des éditeurs de revues (Aliukonis et al., 2020 ; Bonnet et Samama, 2012 ; Decullier et al., 2012 ; Sox et Rennie, 2006).

\section{Conclusion}

Au terme de la présente étude, il est évident que les différents domaines de recherche comportent leurs propres particularités qui facilitent ou entravent l'intérêt des chercheurs envers le processus de rétractation d'articles scientifiques. Or, il faut considérer que ces spécificités ne protègent pas contre la fraude, puisque celle-ci est présente dans tous les domaines de recherche (Grieneisen et Zhang, 2012 ; Lu et al., 2013 ; Marcus et Oransky, 2020). En ce sens, la section traitant de la discussion a proposé plusieurs pistes d'amélioration qui permettraient de réduire les retombées néfastes de la fraude en éducation, et plus largement en sciences humaines : l'implantation de pratiques uniformes quant aux rétractations chez les éditeurs, la diffusion des pratiques éthiques attendues dans les universités et les équipes de recherche, et un accompagnement plus soutenu des étudiants au niveau de l'écriture scientifique. La présente étude comporte toutefois certaines limites. En effet, cette étude étant non systématique, il est inévitable que certains articles ayant abordé les rétractations n'aient pas été repérés. De plus, comme l'auteure a réalisé seule ce travail, une revue de littérature systématique aurait nécessité un investissement de temps et de ressources trop important. Cette limite pose également problème en ce qui concerne la vérification des données, puisqu'un seul individu les a extraites. Il aurait été préférable que plus d'une personne analyse le corpus et que les données extraites fassent l'objet d'un accord interjuges. Par ailleurs, les résultats de cette étude sont limités aux bases de données accessibles à l'aide d'un identifiant de l'Université du Québec à Montréal, ainsi que par l'accès limité aux articles gratuits. De la même façon, l'étude a exploré uniquement les articles rédigés en anglais et en français, et a utilisé un ensemble précis de mots-clés qui pourraient être bonifiés. 
Finalement, il faut considérer que les conclusions dégagées et rapportées au domaine de l'éducation proviennent du champ plus large des sciences humaines et sociales en raison de l'absence d'études sur le sujet en éducation. En ce sens, des particularités du champ de l'éducation pourraient ne pas avoir été prises en compte, ce qui limite la portée des suggestions d'amélioration des pratiques éthiques. Ainsi, d'autres études sont nécessaires, spécifiquement en éducation, pour examiner le phénomène des rétractations de façon plus étendue et bonifier notre compréhension de la fraude dans ce domaine. 


\section{Références}

Aliukonis, V., Poškutè, M. et Gefenas, E. (2020). Perish or publish dilemma: Challenges to responsible authorship. Medicina, 56(3), 123-133. https://doi.org/10.3390/ $\underline{\text { medicina56030123 }}$

Alrawadieh, Z. et Zareer, A. (2019). Exploring retraction in tourism and hospitality journals. European Journal of Tourism Research, 22, 20-30. https://ejtr.vumk.eu/ index.php/about/article/view/372

Aspura, M. K. Y. I., Noorhidawati, A. et Abrizah, A. (2018). An analysis of Malaysian retracted papers: Misconduct or mistakes? Scientometrics, 115(3), 1315-1328. https://doi.org/10.1007/s11192-018-2720-z

Azoulay, P., Bonatti, A. et Krieger, J. L. (2017). The career effects of scandal: Evidence from scientific retractions. Research Policy, 46(9), 1552-1569. https://doi. org/10.1016/j.respol.2017.07.003

Bakker, C. et Riegelman, A. (2018). Retracted publications in mental health literature: Discovery across bibliographic platforms. Journal of Librarianship and Scholarly Communication, 6(General Issue), article eP2199. https://doi. org/10.7710/2162-3309.2199

Basken, P. (2012, 8 janvier). Surge in journal retractions may mask decline in actual problems. Chronicle of Higher Education, 58(19), A12. https://www.chronicle. com/article/surge-in-journal-retractions-may-mask-decline-in-actual-problems/

Benneworth, P. et Jongbloed, B. W. (2010). Who matters to universities? A stakeholder perspective on humanities, arts and social sciences valorisation. Higher Education, 59(5), 567-588. https://doi.org/10.1007/s10734-009-9265-2

Berland, N., Piot, C. et Capkun, V. (2015). Le retrait d'articles en sciences comptables. Comptabilité Contrôle Audit, 21(2), 5-12. https://doi.org/10.3917/cca.212.0005

Bilbrey, E., O’Dell, N. et Creamer, J. (2014). A novel rubric for rating the quality of retraction notices. Publications, 2(1), 14-26. https://doi.org/10.3390/ publications 2010014

Blais, M. et Martineau, S. (2006). L'analyse inductive générale : description d'une démarche visant à donner un sens à des données brutes. Recherches qualitatives, 
26(2), 1-18. http://www.recherche-qualitative.qc.ca/documents/files/revue/ edition_reguliere/numero26\%282\%29/blais_et martineau_final2.pdf

Bonnet, F. et Samama, C. M. (2012). Les cas de fraude dans les publications : de Darsee à Poldermans. La Presse Médicale, 41(9, part 1), 816-820. https://doi. org/10.1016/j.lpm.2012.04.019

Budd, J. M., Coble, Z. C. et Anderson, K. M. (2011, 30 mars-2 avril). Retracted publications in biomedicine: Cause for concern. Dans Association of college and research libraries national conference proceedings (p. 390-395). ACRL 2011, Philadelphie. https://www.ala.org/acrl/sites/ala.org.acrl/files/content/conferences/ confsandpreconfs/national/2011/papers/retracted_publicatio.pdf

Chen, C., Hu, Z., Milbank, J. et Schultz, T. (2013). A visual analytic study of retracted articles in scientific literature. Journal of the American Society for Information Science and Technology, 64(2), 234-253. https://doi.org/10.1002/asi.22755

Cokol, M., Ozbay, F. et Rodriguez-Esteban, R. (2008). Retraction rates are on the rise. EMBO Reports, 9(1), 2. https://doi.org/10.1038/sj.embor.7401143

Committee on Publication Ethics (COPE). (2021). Promoting integrity in scholarly research and its publication. Repéré le 27 janvier 2021, au https:// publicationethics.org/

Coudert, F.-X. (2019). Correcting the scientific record: Retraction practices in chemistry and materials science. Chemistry of Materials, 31(10), 3593-3598. https://doi. org/10.1021/acs.chemmater.9b00897

Cox, A., Craig, R. et Tourish, D. (2018). Retraction statements and research malpractice in economics. Research Policy, 47(5), 924-935. https://doi.org/10.1016/j. respol.2018.02.016

Craig, R., Cox, A., Tourish, D. et Thorpe, A. (2020). Using retracted journal articles in psychology to understand research misconduct in the social sciences: What is to be done? Research Policy, 49(4), article 103930. https://doi.org/10.1016/j. respol.2020.103930

Da Silva, J. A. T. et Bornemann-Cimenti, H. (2017). Why do some retracted papers continue to be cited? Scientometrics, 110(1), 365-370. https://doi.org/10.1007/ $\underline{\text { s11192-016-2178-9 }}$ 
Decullier, E., Huot, L., Samson, G. et Maisonneuve, H. (2013). Visibility of retractions: A cross-sectional one-year study. BMC Research Notes, 6(238), 1-6. https://doi. org/10.1186/1756-0500-6-238

Decullier, E., Samson, G. et Huot, L. (2012). Rétractations pour erreur et pour fraude. La Presse Médicale, 41(9, Part 1), 847-852. https://doi.org/10.1016/j. 1pm.2012.05.006

Fanelli, D. (2009). How many scientists fabricate and falsify research? A systematic review and meta-analysis of survey data. PLOS ONE, 4(5), article e5738. https:// doi.org/10.1371/journal.pone.0005738

Fanelli, D., Costas, R. et Larivière, V. (2015). Misconduct policies, academic culture and career stage, not gender or pressures to publish, affect scientific integrity. PLoS ONE, 10(6), article e0127556. https://doi.org/10.1371/journal.pone.0127556

Fang, F. C. et Casadevall, A. (2011). Retracted science and the retraction index. Infection and Immunity, 79(10), 3855-3859. https://doi.org/10.1128/IAI.05661-11

Funk, C. L., Barrett, K. A. et Macrina, F. L. (2007). Authorship and publication practices: Evaluation of the effect of responsible conduct of research instruction to postdoctoral trainees. Accountability in Research, 14(4), 269-305. https://doi. org/10.1080/08989620701670187

George, S. L. (2016). Research misconduct and data fraud in clinical trials: Prevalence and causal factors. International Journal of Clinical Oncology, 21(1), 15-21. https://doi.org/10.1007/s10147-015-0887-3

Grieneisen, M. L. et Zhang, M. (2012). A comprehensive survey of retracted articles from the scholarly literature. PLoS ONE, 7(10), 1-15. https://doi.org/10.1371/journal. pone. 0044118

Hille, K. (2011). Bringing research into educational practice: Lessons learned. Mind, Brain, and Education, 5(2), 63-70. https://doi. org/10.1111/j.1751-228X.2011.01111.X

Hu, G., Yang, Y. et Tang, L. (2019). Retraction and research integrity education in China. Science and Engineering Ethics, 25(1), 325-326. https://doi.org/10.1007/ $\underline{\text { s11948-017-0017-x }}$ 
Karabag, S. F. et Berggren, C. (2012). Retraction, dishonesty and plagiarism: Analysis of a crucial issue for academic publishing, and the inadequate responses from leading journals in economics and management disciplines. Journal of Applied Economics and Business Research, 2(3), 172-183. http://liu.diva-portal.org/ smash/get/diva2:582178/FULLTEXT01.pdf

Karabag, S. F. et Berggren, C. (2016). Misconduct, marginality and editorial practices in management, business and economics journals. PLoS ONE, 11(7), article e0159492. https://doi.org/10.1371/journal.pone.0159492

Lambert-Chan, M. (2013, 7 août). Des co-auteurs d'articles rétractés voient leur carrière compromise. Affaires universitaires. https:// www.affairesuniversitaires.ca/actualites/actualites-article/ des-coauteurs-darticles-retractes-voient-leur-carriere-compromise/

Lee, C.-S. et Schrank, A. (2010). Incubating innovation or cultivating corruption? The developmental state and the life sciences in Asia. Social Forces, 88(3), 12311255. http://dx.doi.org/10.1353/sof.0.0282

Lei, L. et Zhang, Y. (2018). Lack of improvement in scientific integrity: An analysis of WoS retractions by Chinese researchers (1997-2016). Science and Engineering Ethics, 24(5), 1409-1420. https://doi.org/10.1007/s11948-017-9962-7

Li, G., Kamel, M., Jin, Y., Xu, M. K., Mbuagbaw, L., Samaan, Z., Levine, M. A. et Thabane, L. (2018). Exploring the characteristics, global distribution and reasons for retraction of published articles involving human research participants: a literature survey. Journal of Multidisciplinary Healthcare, 11, 39-47. https://doi. org/10.2147/JMDH.S151745

Lu, S. F., Jin, G. Z., Uzzi, B. et Jones, B. (2013). The retraction penalty: Evidence from the web of science. Scientific Reports, 3(1), article 3146. https://doi.org/10.1038/ $\underline{\text { srep03146 }}$

Madlock-Brown, C. R. et Eichmann, D. (2015). The (lack of) impact of retraction on citation networks. Science and Engineering Ethics, 21(1), 127-137. https://doi. org/10.1007/s11948-014-9532-1

Marcus, A. et Oransky, I. (2011). The paper is not sacred. Nature, 480(7378), 449-450. https://doi.org/10.1038/480449a 
Marcus, A. et Oransky, I. (2020). Retraction Watch. Repéré le 16 juillet 2020, au https:// retractionwatch.com/

Mena, J. D., Ndoye, M., Cohen, A. J., Kamal, P. et Breyer, B. N. (2019). The landscape of urological retractions: The prevalence of reported research misconduct. BJU International, 124(1), 174-179. https://doi.org/10.1111/bju.14706

Mongeon, P. et Larivière, V. (2016). Costly collaborations: The impact of scientific fraud on co-authors' careers. Journal of the Association for Information Science and Technology, 67(3), 535-542. https://doi.org/10.1002/asi.23421

Nath, S. B., Marcus, S. C. et Druss, B. G. (2006). Retractions in the research literature: Misconduct or mistakes? Medical Journal of Australia, 185(3), 152-154. https:// doi.org/10.5694/j.1326-5377.2006.tb00504.x

Nature. (2013). Seven days: 26 April-2 May 2013. Nature News, 497(7447), 10-11. https://doi.org/10.1038/497010a

Nogueira, T. E., Gonçalves, A. S., Leles, C. R., Batista, A. C. et Costa, L. R. (2017). A survey of retracted articles in dentistry. BMC Research Notes, 10(1), article 253. https://doi.org/10.1186/s13104-017-2576-y

Palla, I. A., Singson, M. et Thiyagarajan, S. (2020). A comparative analysis of retracted papers in Health Sciences from China and India. Accountability in Research, 27(7), 401-416. https://doi.org/10.1080/08989621.2020.1754804

Peterson, G. M. (2013). Characteristics of retracted open access biomedical literature: A bibliographic analysis. Journal of the American Society for Information Science and Technology, 64(12), 2428-2436. https://doi.org/10.1002/asi.22944

QSR International. (2020). NVivo (version 12) [Logiciel]. QSR International Pty Ltd. https://www.qsrinternational.com/nvivo-qualitative-data-analysis-software/home

Randi, J. et Corno, L. (2007). Theory into practice: A matter of transfer. Theory Into Practice, 46(4), 334-342. https://doi.org/10.1080/00405840701593923

Rosenkrantz, A. B. (2016). Retracted publications within radiology journals. American Journal of Roentgenology, 206(2), 231-235. https://doi.org/10.2214/ $\underline{\text { AJR. } 15.15163}$ 
Rubbo, P., Helmann, C. L., Bilynkievycz dos Santos, C. et Pilatti, L. A. (2019).

Retractions in the engineering field: A study on the web of science database.

Ethics \& Behavior, 29(2), 141-155. https://doi.org/10.1080/10508422.2017.1390 $\underline{667}$

Shuai, X., Rollins, J., Moulinier, I., Custis, T., Edmunds, M. et Schilder, F. (2017). A multidimensional investigation of the effects of publication retraction on scholarly impact. Journal of the Association for Information Science and Technology, 68(9), 2225-2236. https://doi.org/10.1002/asi.23826

Simard, M., Tremblay, M.-E., Lavoie, A. et Audet, N. (2013). Enquête québécoise sur le développement des enfants à la maternelle 2012 : portrait statistique pour le Québec et ses régions administratives. https://bdso.gouv.qc.ca/docs-ken/ multimedia/PB01670FR_enfants_maternelle2012H00F00.pdf

Sox, H. C. et Rennie, D. (2006). Research misconduct, retraction, and cleansing the medical literature: lessons from the Poehlman case. Annals of Internal Medicine, 144(8), 609-613. https://doi.org/10.7326/0003-4819-144-8-200604180-00123

Steen, R. G. (2011). Retractions in the scientific literature: Do authors deliberately commit research fraud? Journal of Medical Ethics, 37(2), 113-117. https://doi. org/10.1136/jme.2010.038125

Steen, R. G. (2012). Retractions in the medical literature: How can patients be protected from risk? Journal of Medical Ethics, 38(4), 228-232. https://jme.bmj.com/ content $/ 38 / 4 / 228$.info

Steneck, N. H. (2006). Fostering integrity in research: Definitions, current knowledge, and future directions. Science and Engineering Ethics, 12(1), 53-74. https://doi. org $/ 10.1007 / \mathrm{p} 100022268$

Stretton, S., Bramich, N. J., Keys, J. R., Monk, J. A., Ely, J. A., Haley, C., Woolley, M. J. et Woolley, K. L. (2012). Publication misconduct and plagiarism retractions: a systematic, retrospective study. Current Medical Research and Opinion, 28(10), 1575-1583. https://doi.org/10.1185/03007995.2012.728131

Tchao, R. (2014). A need to archive correct biomedical scientific data and to prevent continued citation of retracted scientific publications. International Journal of 
Humanities and Arts Computing, 8(supplement), 29-37. https://doi.org/10.3366/ ijhac.2014.0097

Thomas, D. R. (2006). A general inductive approach for analyzing qualitative evaluation data. American Journal of Evaluation, 27(2), 237-246. https://doi. org/10.1177/1098214005283748

U.S. Department of Education, Office of the Deputy Secretary, Planning and Performance Management Service. (2002, mars). U.S. Department of Education Strategic Plan 2002-2007. Washington, DC. https://www2.ed.gov/about/reports/strat/plan200207/plan.pdf

Van Noorden, R. (2011). Science publishing: The trouble with retractions. Nature, 478(7367), 26-28. https://doi.org/10.1038/478026a

Williams, P. et Wager, E. (2013). Exploring why and how journal editors retract articles: findings from a qualitative study. Science and Engineering Ethics, 19(1), 1-11. http://dx.doi.org/10.1007/s11948-011-9292-0

Yoshikawa, H., Weiland, C., Brooks-Gunn, J., Burchinal, M. R., Espinosa, L. M., Gormley, W. T., Ludwig, J., Magnuson, K. A., Phillips, D. et Zaslow, M. J. (2013, octobre). Investing in our future: The evidence base on preschool education. Society for Research in Child Development. https://www.fcd-us.org/ assets/2016/04/Evidence-Base-on-Preschool-Education-FINAL.pdf 